\title{
Die Geschichte des djb als Chronik der Emanzipation
}

\section{Gerda Hasselfeldt MdB \\ Vizepräsidentin des Deutschen Bundestags, Berlin}

Sehr geehrte Frau Präsidentin Wagner,

sehr geehrte Damen und Herren,

ich begrüße Sie im großen Protokollsaal hier im Reichstagsgebäude und darf Ihnen zu Ihrem 60-jährigen Jubiläum auch die herzlichen Grüße und Glückwünsche des Präsidenten des Deutschen Bundestages, Prof. Norbert Lammert, übermitteln.

Vor gut hundert Jahren, so hat es der Bayerische Rundfunk einmal dokumentiert, wurde in diesem Haus, dem Deutschen Reichstag, folgendes erklärt: „Alles an der Frau ist zierlicher und kleiner, damit der Mann sie beschütze. Die Stimme der Frau taugt ja schon gar nicht zu politischen Reden, weil die Stimmritze der Frau viel kleiner ist und nicht das nötige Volumen hat."

Schon damals gab es mutige Frauen, die sofort konterten und zu Recht den kausalen Zusammenhang zwischen Stimmritze und Stimmrecht bestritten. Und doch dauerte es noch 25 Jahre, bis das allgemeine Wahlrecht für Frauen eingeführt wurde. Heute liegt der Frauenanteil im Deutschen Bundestag immerhin bei einem Drittel - und glücklicherweise hat sich mittlerweile auch die Auffassung durchgesetzt, Frauen in aller Regel nicht nach dem Volumen ihrer Stimme, sondern nach ihren Talenten, Fähigkeiten und Leistungen zu beurteilen.

Eine ganz ähnliche Entwicklung vollzog sich auch im juristischen Berufsstand. Zu Beginn des 20. Jahrhunderts waren Frauen noch gar nicht überall zum Hochschulstudium zugelassen. Erst in der Weimarer Republik konnten sie in den juristischen Staatsdienst eintreten, was ihnen aber bereits wenige Jahre später im nationalsozialistischen Deutschland wieder untersagt wurde. 1939 gab es keine Richterinnen, keine Staatsanwältinnen und ganze neun Rechtsanwältinnen in Deutschland.

Heute gibt es mehr Frauen als Männer in den juristischen Studiengängen und eine große Zahl sehr erfolgreicher Frauen

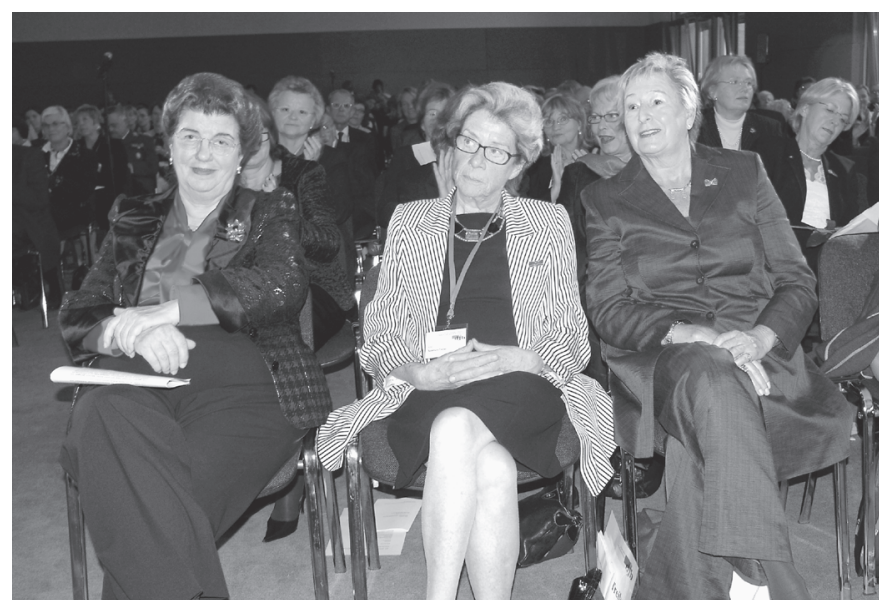

in den juristischen Berufen. An diesem langen Prozess haben viele mitgewirkt. Der Deutsche Juristinnenbund, der heute sein 60-jähriges Bestehen feiert, hat zweifellos auch dazu einen wichtigen Beitrag geleistet.

Dass das Engagement des Juristinnenbundes dabei nicht nur für Frauen wertvoll war, sondern auch ingesamt bedeutsam, steht fest. Denn es ist mittlerweile unbestritten, dass die Qualität der Arbeit eines Gremiums besser ist, wenn nicht nur Frauen und nicht nur Männer, sondern beide gleichberechtigt zusammenarbeiten und auch Frauen Führungspositionen innehaben. Dass Frauen hier auch heute noch deutlich unterrepräsentiert sind, ist nicht zuletzt deshalb ein Problem: Frauen bringen ihre eigene Sicht und ihre eigenen Talente ein - und je vielfältiger die Talente, desto besser das Ergebnis.

Der Deutsche Bundestag ist der Bitte des Juristinnenbundes, seine Jubiläumsfeier zum 60-jährigen Bestehen im Reichstagsgebäude abhalten zu können, sehr gerne nachgekommen. Schließlich ist uns Parlamentariern der djb als ein unabhängiger und kritischer Fachverband ein unverzichtbarer Ratgeber bei der Gesetzgebung. Er war und ist wichtiger Motor für die stetige Weiterentwicklung des Rechts, insbesondere im Bereich der Gleichberechtigung und der Gleichstellung von Frauen.

Der Deutsche Juristinnenbund steht in kontinuierlichem Kontakt zu den Berichterstattern der Fraktionen, er beteiligt sich an Anhörungen in den Ausschüssen und er ist persönlicher Ansprechpartner für einzelne Abgeordnete. Die Vielfalt der Themen, zu denen der djb Stellung bezieht, ist dabei beeindruckend: Sie reichen vom Unterhaltsrecht über die Problematik der gleichen Bezahlung bis hin zum Stalking. Die zunehmende Bedeutung der Rechtsetzung auf europäischer Ebene macht das Aufgabenspektrum noch vielfältiger.

Doch nicht nur für den Gesetzgeber stellt der djb eine Bereicherung dar, sondern auch für die Gesellschaft. Der Juristinnenbund hat sich nie davor gescheut, an wissenschaftlichen und öffentlichen Diskussionen mitzuwirken und sich einzumischen. Schon 1948, im Gründungsjahr des Deutschen Juristinnenbundes, war es mit Elisabeth Selbert eine Mitstreiterin des djb der ersten Stunde, die die Aufnahme des Gleichberechtigungsgrundsatzes ins Grundgesetz durchsetzte. Mittlerweile sind viele Mitglieder des Juristinnenbundes an wichtigen Schaltstellen tätig, in Politik, Verwaltung, Rechtsprechung, in Wirtschaft und Wissenschaft. Sie führen dort das fort, was Elisabeth Selbert im Parlamentarischen Rat begonnen hat: Sie

$\varangle$ In der ersten Reihe (v.I.n.r.): Rechtsanwältin Dr. Lore Maria Peschel-Gutzeit, Senatorin a.D., Berlin, Präsidentin des BPatG i.R. Antje Sedemund-Treiber, Bonn und Rechtsanwältin Renate Damm, Hamburg (alle drei Ehrenpräsidentinnen des djb). 
setzen sich in ihren jeweiligen Aufgaben für Frauenförderung ein - und weisen auf noch bestehende Defizite wie etwa die Entgeltdiskriminierung hin.

Die Geschichte des Deutschen Juristinnenbundes liest sich als eine Chronik der Emanzipation in der Bundesrepublik. Dass bis heute vieles erreicht wurde, kommt nicht nur darin zum Ausdruck, dass das Bundesjustizministerium seit 1992 mit nur zweijähriger Unterbrechung immer von einer Frau geleitet wurde. Auch der Blick auf die Gäste der heutigen Veranstaltung mit den zahlreichen herausragenden Vertreterinnen aus Justiz, Wissenschaft, Wirtschaft und Politik zeigt, dass der djb seit Jahrzehnten erfolgreiche Arbeit leistet.

$\mathrm{Zu}$ guter Letzt bietet der Deutsche Juristinnenbund etwas, das gerade für uns Frauen, die wir oft zum Einzelkämpfertum neigen, besonders wertvoll ist: eine Plattform für den Austausch von Wissen und Erfahrungen, ein Netzwerk für Frauen an den unterschiedlichsten Positionen, aber mit verwandten Tätigkeiten. Dies ist nicht nur eine Bereicherung für jedes einzelne Mitglied des djb, sondern es fördert auch die Arbeit des gesamten Verbandes.

Ihre Arbeit ist nach 60 Jahren nicht zu Ende. Wir brauchen den djb auch weiterhin - in der Gesetzgebung als kompetenten Berater, in der gesellschaftspolitischen Diskussion als kritischen Impulsgeber. Und die Frauen brauchen Sie in der täglichen Praxis als wertvolles Netzwerk.

In diesem Sinne danke ich allen Verantwortlichen des Verbandes für Ihre engagierte Arbeit und wünsche Ihnen weiterhin eine glückliche Hand und viel Erfolg.

\title{
Lasten und Chancen gerecht verteilen
}

\author{
Brigitte Zypries MdB

\section{Bundesministerin der Justiz, Berlin}

Sehr geehrte Festgesellschaft, das waren bewegte Zeiten - damals im Sommer 1948: In Westdeutschland führten die Alliierten die D-Mark ein und hier in Berlin begann die Blockade durch die Sowjets. Der Parlamentarische Rat wurde nach Bonn einberufen, und es war klar, dass jetzt die politischen Weichen für das neue Deutschland gestellt wurden. Die Gründung des Deutschen Juristinnenbundes erfolgte genau in der richtigen Zeit. Tatsächlich gab es schon wenige Wochen später eine Menge zu tun für den neu gegründeten Verband. Wenn es nämlich allein nach den Männern im Parlamentarischen Rat gegangen wäre, dann hätte das Grundgesetz uns Frauen nur das Wahlrecht und einige andere staatsbürgerlichen Rechte garantiert. Es war Elisabeth Selbert, Rechtsanwältin aus Kassel und Mitglied des djb, die dann den Satz formuliert hat, der heute in Artikel 3 steht: „Männer und Frauen sind gleichberechtigt.“ Diese Gründungsgeschichte zeigt, dass der djb weit mehr ist als eine Berufsorganisation. Er ist seit sechs Jahrzehnten ein wichtiger Mitstreiter im Kampf für die Rechte der Frauen und gerade als Justizministerin habe ich auch persönlich oft Unterstützung vom djb bekommen. Dieser Rückenwind ist politisch wichtig und er tut manchmal auch persönlich ganz gut, und deshalb gilt mein großer Dank allen Frauen, die sich im djb engagiert haben und dies bis heute tun.

Meine Damen,

allerdings wissen wir, dass es mit der tatsächlichen Gleichstellung bis heute eine schwierige Sache ist. Die Zulassung der Frauen zum Richteramt musste etwa Anfang der 20er Jahre gegen den vehementen Widerstand der Konservativen und der Juristenverbände durchgesetzt werden. Noch bis in die 50er Jahren galt zudem die Zölibatsklausel im Beamtenrecht. Wenn eine Beamtin heiratete, konnte sie kurzerhand entlassen wer-

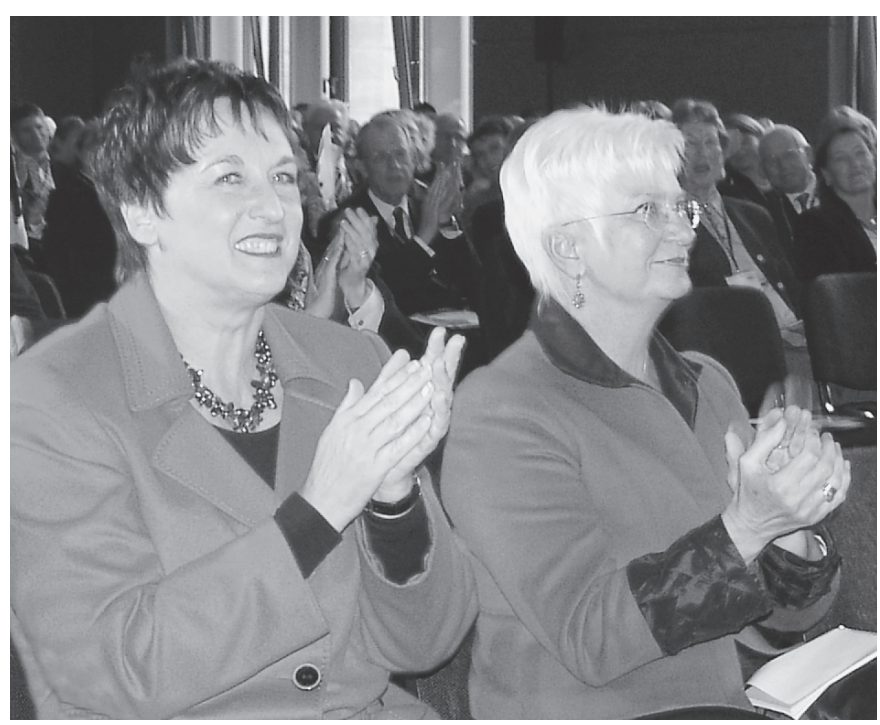

$\triangle$ Bundesjustizministerin Brigitte Zypries MdB (links) und Vizepräsidentin des Deutschen Bundestags Gerda Hasselfeldt MdB.

den, wenn - so hieß das damals - „ihre wirtschaftliche Versorgung dauernd gesichert ist“. Es ist auch ein Verdienst des djb und der Arbeit seiner ersten Jahre, dass diese diskriminierenden Vorschriften abgeschafft worden sind. In vielen Rechtsgebieten hat es zudem bis in die 70er Jahre gedauert, Benachteiligungen von Frauen abzuschaffen, zum Beispiel im Familienrecht. Der Versorgungsausgleich nach einer Scheidung wurde erst 1976 eingeführt, und mein damaliger Amtsvorgänger Hans-Jochen Vogel erzählt, dass sich bei ihm bis heute gelegentlich Männer bitter beklagen, dass sie seither ihre Rentenansprüche mit ihren geschiedenen Ehefrauen teilen müssen. Die Benachteiligung im 